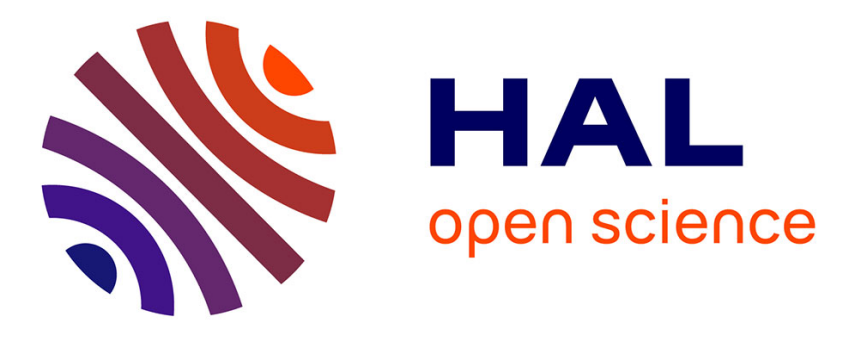

\title{
Micro-fabricated Surface Electrode Ion Trap with 3D-TSV Integration for Scalable Quantum Computing
}

\author{
Jing Tao, Luca Guidoni, Hong Yu Li, Lin Bu, Nam Piau Chew, Chuan Seng
}

Tan

\section{- To cite this version:}

Jing Tao, Luca Guidoni, Hong Yu Li, Lin Bu, Nam Piau Chew, et al.. Micro-fabricated Surface Electrode Ion Trap with 3D-TSV Integration for Scalable Quantum Computing. 2019 Electron Devices Technology and Manufacturing Conference (EDTM), Mar 2019, Singapore, France. pp.300-302, 10.1109/EDTM.2019.8731188 . hal-03060092

\section{HAL Id: hal-03060092 \\ https://hal.science/hal-03060092}

Submitted on 13 Dec 2020

HAL is a multi-disciplinary open access archive for the deposit and dissemination of scientific research documents, whether they are published or not. The documents may come from teaching and research institutions in France or abroad, or from public or private research centers.
L'archive ouverte pluridisciplinaire HAL, est destinée au dépôt et à la diffusion de documents scientifiques de niveau recherche, publiés ou non, émanant des établissements d'enseignement et de recherche français ou étrangers, des laboratoires publics ou privés. 


\title{
Micro-fabricated Surface Electrode Ion Trap with 3D-TSV Integration for Scalable Quantum Computing
}

\author{
Jing Tao ${ }^{1}$, Luca Guidoni ${ }^{2}$, Hong Yu Li ${ }^{3}$, Lin Bu ${ }^{3}$, Nam Piau Chew ${ }^{1}$ and Chuan Seng $\operatorname{Tan}^{1 *}$ \\ ${ }^{1}$ School of Electrical and Electronic Engineering, Nanyang Technological University, Singapore \\ 639798 \\ ${ }^{2}$ Laboratoire Matériaux et Phénomènes Quantiques, Université Paris Diderot, France, 75205 \\ ${ }^{3}$ Institute of Microelectronics, Agency for Science, Technology and Research (A*STAR), \\ Singapore 117685 \\ Email: tancs@ntu.edu.sg
}

\begin{abstract}
In this paper, 3D architecture for TSV integrated Si surface ion-trap is proposed, in which the TSV and microbump technology is used to connect the surface electrodes of ion trap to a bottom Si interposer. The pseudopotential simulation is used to determine the trapped ion height for both the "planar trap" and "TSV trap" geometry. No significant deviation in the pseudopotential for both cases is observed. Preliminary micro-fabricated ion-trap chips are characterized. The proposed technology is promising in form factor and parasitic reduction of micro-fabricated surface ion-trap for scalable quantum computing application.
\end{abstract}

(Keywords: surface ion trap, 3D TSV integration, quantum computing)

\section{Introduction}

Quantum computing is widely touted as one of the most likely technologies to sustain the future demand for high performance computing. A promising approach to realize quantum computer is to utilize the atomic ions suspended in vacuum as quantum bits (qubits) to perform quantum operations [1]. The ions are confined in free space by a group of surface electrodes which generate static (DC) and radio-frequency (RF) electric fields above the electrode surface. Laser beams with the appropriate wavelengths are used to cool the ions to the ground vibrational energy state and to perform qubit operations by addressing the electron energy states of the ions. The modern ion trap chips facilitate a large number of multi-segmented surface electrodes fabricated on Si substrate to manipulate high density ion arrays or form multiple ion trapping zones [2].

One of the key challenge of the ion trapping technology is to interconnect the ever increasing electrode number to the outer DC/RF power source in a scalable way. The conventional wire-bonding method requires a space-consuming peripheral bonding pads design on the chip surface area and it also has the drawback as obstruction to laser incident from the periphery of the chip to the trapped ions. With the advanced 3D integration technologies, the ion trap chip is proposed to be vertically stacked on a Si interposer, where the through-silicon via (TSV) and micro-bump are utilized to form the vertical interconnects to connect the surface electrodes. Fig. 1 shows the schematic of the proposed TSV integrated ion trap die stacked on a Si interposer, where an ion is trapped above the trap chip surface. The proposed architecture presents a miniaturized ion trap system with the advantage of high density electrode integration capability, smaller RC delay, compact form factor and clear accessibility for laser beam on the chip surface. 


\section{Pseudopotential Simulation}

The design of surface electrode ion trap is based on 5-wire design, with a typical surface geometry (planar structure with wire-bonding pads) shown in Fig. 2 (a), where two RF lines are arranged parallel to a center DC line and the segmented DC electrodes are located on each side of two RF lines. By applying a RF drive voltage, a pseudopotential well is generated to trap the ion radially. DC voltages are applied to segmented DC electrodes to confine the ion axially. The central DC line is mainly used to compensate ion micromotion. By TSV integration, the surface geometry of the trap can be reduced by removing the wire-bonding pads in Fig. 2 (a). TSV trap geometry is shown in Fig. 2 (b), with a form factor reduction of $3 x$ compared to the corresponding planar trap geometry. RF pseudopotential is simulated using finite element method (FEM) with a commercial software [3]. The simulation is performed for an $88 \mathrm{Sr}+$ ion with a RF drive voltage of $200 \mathrm{~V}, 56 \mathrm{MHz}$. Fig. 2 (c) shows the simulated pseudopotential contour, where a symmetric pseudopotential well is generated to trap the ion above the center of the trap surface (xy-plane). The pseudopotential along on ion height direction (z-axis) is plotted in Fig. 2 (d). The simulated ion height is $\sim 40 \mu \mathrm{m}$ above trap surface with a trap depth of $\sim 300 \mathrm{meV}$. Simulation shows no obvious difference of ion height and trap depth between the planar and TSV trap geometry.

\section{TSV Trap Fabrication}

The planar trap (without TSV) is firstly fabricated using an innovative approach which is completely compatible with CMOS back-end processes. The trap is fabricated in a high resistivity $(>750 \Omega \cdot \mathrm{cm})$ Si substrate with a layer of $3 \mu \mathrm{m}$ thick SiO2 to reduce the substrate induced RF loss. The trap electrode is composed of $3 \mu \mathrm{m}$ thick $\mathrm{Cu}$ and $0.2 \mu \mathrm{m}$ Au finish to form a thick metal layer to withstand high RF voltage ( $200 \mathrm{~V})$. The oxide in the electrode gap area is etched, in order to minimize the line of sight access to the ion from exposed dielectrics, thereby reducing the impact of stray electric charges [4]. The fabricated planar trap is shown in Fig. 3 (a) and (b) with the top view of the geometry surface and crosssectional view of the electrode and oxide layer. The breakdown voltage of the trap die is measured by voltage-stressing method. The trap has a leakage current in the order of 10-10 A under a $200 \mathrm{~V}$ static bias, which is comparable to reported data [5]. The capacitance induced by the electrodes and the Si substrate is measured by Capacitance-Voltage method and is in the order of 10-12 F, which is comparable to reported data [6]. The major process steps for TSV trap chip fabrication include TSV, Front Side (FS) redistribution layer (RDL), via-revealing and backside Underbump Metallisation (UBM) and backside microbump plating. Bosch process is used to form the TSV trench of $20 \mu \mathrm{m}$ diameter and $100 \mu \mathrm{m}$ depth followed by TSV liner deposition and Cu electrochemical plating process (ECP) to fill the TSV. Cu ECP is also used to form FS RDL as the trap electrodes. The inter-electrode oxide layer is removed by dry etch method. For backside fabrication, the wafer is thinned to reveal vias followed by oxide deposition and chemical-mechanical polishing (CMP) process. BS UBM and microbump are fabricated by $\mathrm{Cu}$ ECP and polyimide (PI) passivation method as reported in [7] . The TSV trap is stacked on Si interposer die using microbump bonding. In interposer die, $\mathrm{Cu}$ RDL is designed to redistribute the trap circuit to the outer DC/RF supply by wire-bonding through CPGA package. A preliminary fabrication of TSV structure is demonstrated in Fig. 3 (c), where cross-sectional view of Cu fill TSV with 20 $\mu \mathrm{m}$ diameter and $100 \mu \mathrm{m}$ depth is shown.

Conclusion 
3D architecture for TSV integrated Si surface ion trap is proposed is this work. The pseudopotential simulation shows no obvious difference of ion height and trap depth between the planar and TSV trap geometry. The CMOS-compatible planar and TSV trap fabrication process indicates the large scale manufacturing and integration capability of the microfabricated surface traps for the complex quantum computing system.

\section{Acknowledgments}

Authors are grateful for funding support from A*STAR Quantum Technology for Engineering (A1685b0005).

\section{References}

[1] C. R. Monroe, R. J. Schoelkopf, and M. D. Lukin, "Quantum Connections," Sci. Am., vol. 314, no. 5, pp. 50-57, 2016.

[2] D. L. Moehring et al., "Design, fabrication and experimental demonstration of junction surface ion traps," New J. Phys., vol. 13, 2011.

[3] "Https://www.comsol.com/." .

[4] D. Stick et al., "Demonstration of a microfabricated surface electrode ion trap," pp. 1-4, 2010.

[5] K. K. Mehta et al., "Ion traps fabricated in a CMOS foundry," vol. 1749011749, no. 2015, 2016.

[6] S. C. Doret et al., "Controlling trapping potentials and stray electric fields in a microfabricated ion trap through design and compensation," New J. Phys., vol. 14, 2012. [7] S. C. Chong, H. Li, L. Xie, V. N. Sekhar, and D. I. Cereno, "Development of Chip on wafer Bonding with Non Conductive Film using Gang Bonder," pp. 1-5, 2017.

Fig. 1. Schematic of 3D architecture of surface electrode ion trap with TSV interconnects and stacked to a Si interposer using micro-bumps

Fig. 2. FEM simulation of RF pseudopotential for the respective trap geometry of (a) planar trap and (b) TSV trap; (c) shows the pseudopotential contour created by the TSV trap geometry, where $\mathrm{x}=0$ is the center of the trap surface and $\mathrm{y}$ is the height above the trap surface. The legend shows pseudopotential value in meV. (d) compares pseudopotential plot of the planar and TSV trap. The simulated ion height is $\sim 40 \mu \mathrm{m}$ above the trap surface for both trap geometries. The simulation is performed for an $88 \mathrm{Sr}+$ ion with a RF drive voltage of $200 \mathrm{~V}, 56 \mathrm{MHz}$.

Fig. 3. Planar and TSV trap fabrication: (a) optical image of planar trap surface, (b) SEM crosssectional view of the electrode and etched oxide gap and (c) SEM image of Cu filled TSV of $20 \mu \mathrm{m}$ diameter and $100 \mu \mathrm{m}$ depth. 

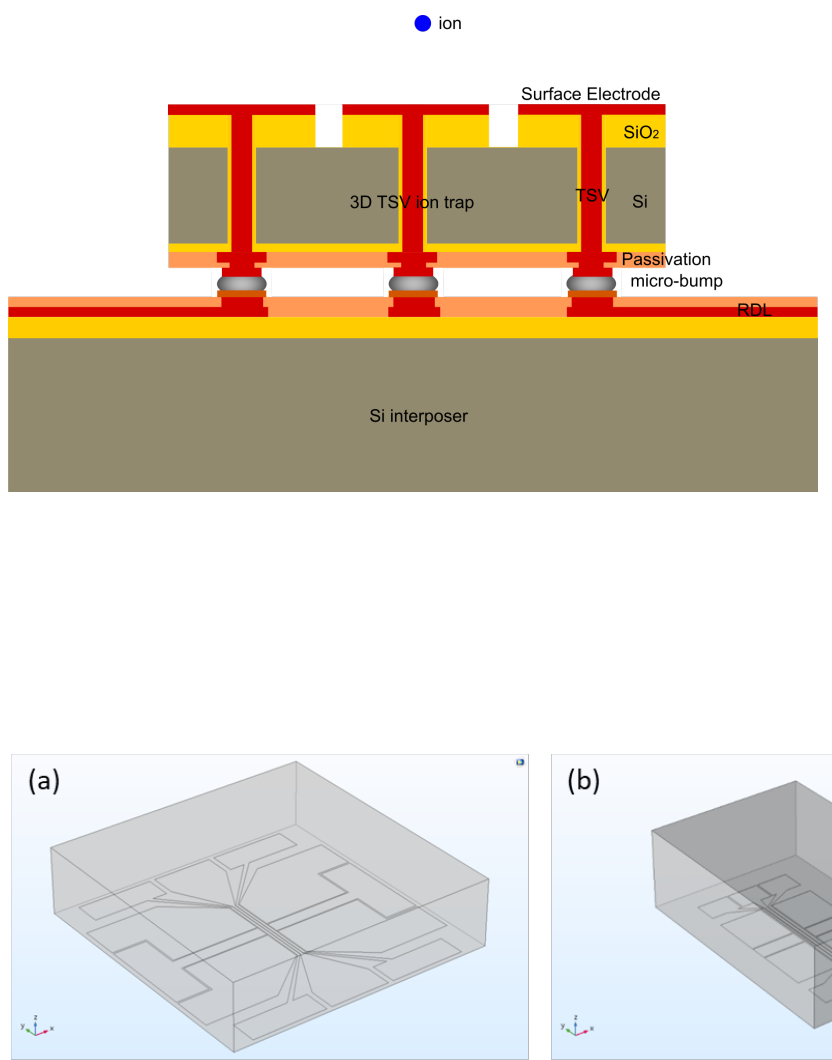

(b)
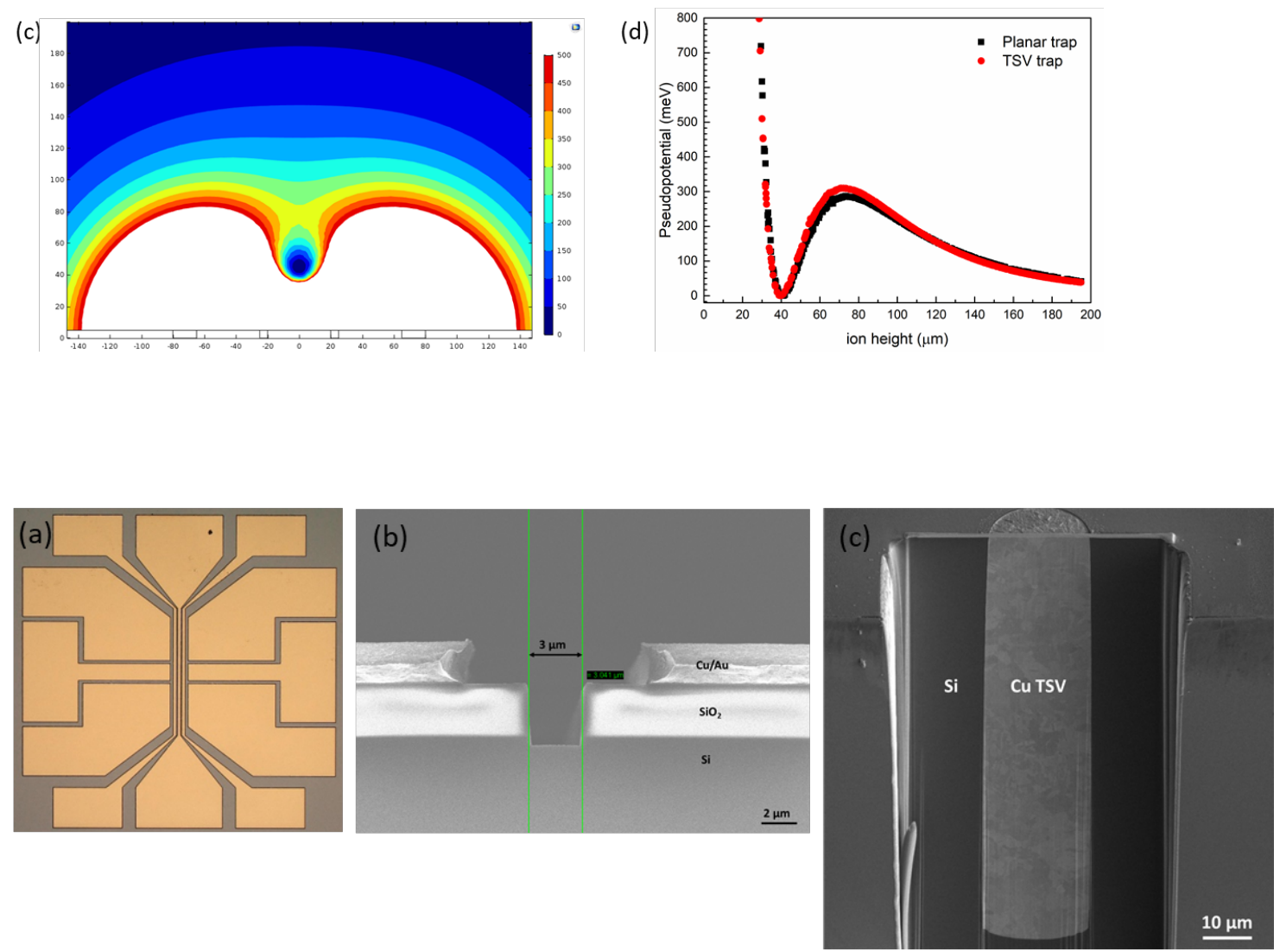